\title{
Recent Updates on Intravenous Thrombolysis and Endovascular Theraphy for Hyperacute Manegement of Ischemic Strokes
}

\author{
Rocco Galimi* \\ Department of Neurology, Local Health Unit of Valtellina and Alto Lario, Sondalo Hospital, Sondrio (Italy)
}

${ }^{\star}$ Corresponding author: Rocco Galimi, Department of Neurology, Local Health Unit of Valtellina and Alto Lario, Sondalo Hospital, Sondrio (Italy); E-mail: rocco.galimi@asst-val.it

Received: September 03, 2021; Accepted: September 16, 2021; Published: October 01, 2021

\begin{abstract}
Acute ischemic stroke (IAS) care has recently been revolutionized with the advent of acute reperfusion therapies. During recent decades, a growing number of randomized controlled clinical trials (RCTs) have helped expand a set of tools for emergency management of acute ischemic stroke, specially advanced imaging that has allowed the expansion of the therapeutic window by various mismatch assessments. Currently two available reperfusion therapies that have been shown to improve outcome in AIS patients, intravenous thrombolysis (IVT) and endovascular therapy (EVT), scilicet mechanical clot removal, both of which are highly time dependent. They have been shown to improve outcome in patients with wake-up strokes or symptom onset beyond 4.5 hours for intravenous thrombolysis and beyond 6 hours for endovascular treatment. Next-generation trials are attempting to expand the time window to benefit additional patients who present in the late time window for both intravenous thrombolysis and endovascular reperfusion therapies. However, they require advanced neuroimaging to select stroke patients safely. Modern trials extended the use of IVT and EVT in time windows that have been originally considered dangerous, in increasingly broad categories of patients during an extended time window. Under current guidelines, IVT is used to treat acute stroke only if it can be ascertained that the time since the onset of symptoms was less than 4.5 hours and 6 hours for EVT using standard neuroimaging such as computed tomography for IVT and CT plus CT angiography for EVT. It is true that recent randomized controlled trials (RCTs) have extended the therapeutic time window, however the studies have used different imaging and clinical inclusion criteria for patient selection during the initiation of this new stroke treatment paradigm, enroll patients based on the presence of a target mismatch on multimodal imaging. Furthermore, for those patients in expanded time windows, guidelines now recommend the use of "advanced" imaging techniques in the acute setting, including CT perfusion and MRI, to guide therapeutic decision-making. Advanced imaging such as perfusion-weighted imaging - core mismatch and conventional imaging such as diffusion-weighted image - fluid-attenuated inversion recovery mismatch played a role in expanding the therapeutic window to wake-up strokes or unwitnessed strokes. This article aims to review the current approaches using neuroimaging techniques to expand eligibility for IVT and EVT in acute ischemic stroke patients with stroke of unknown symptom onset, with an emphasis on new updates to qualifying patient populations and time periods for treatment. The current state of AIS reperfusion treatments in extended time windows it comes to us from high quality data from RCTs that used advanced neuroimaging (CT / MRI perfusion or MRI) to select patients.
\end{abstract}

Keywords: advanced neuroimaging, endovascular treatment, intravenous thrombolysis, ischemic stroke, large vessel occlusion, thrombectomy

\section{Introduction}

Acute stroke is an emergency, that may lead to permanent disability and death. Therefore, the foundation of care is based on choosing the optimal treatment option for the patient based on the best scientific evidence. Every year, about 22 million people around the world suffer from stroke [1]. Roughly, $87 \%$ of cerebrovascular events are due to artery occlusion as opposed to hemorrhage [2]. Acute ischemic stroke (AIS), result when a vessel supplying blood to the brain is occluded, and impairs blood flow to part of the brain. When acute cerebral ischemia occurs due to the occlusion of an intracranial artery, the part of the brain that is irreversibly lost (ischemic core) is surrounded by brain parenchyma that can be salvaged (penumbra) if prompt recanalization takes place [2]. In fact, it's so, ischemic penumbra denotes the part of an acute ischemic stroke that is at risk of progressing to infarction but is still salvageable if reperfused. During the last decade, the management of acute ischemic stroke has changed dramatically, from an expectant bedside attitude towards active treatment, thanks to the continuous improvement of new therapeutic options. Neuroimaging information has demonstrated efficacy to visualize effects that neurological examination cannot be seen, such as penumbra, and advanced imaging in stroke has significantly influenced and explored the pathological conditions of the ischemic blood flow. At present intravenous thrombolysis (IVT) and endovascular therapy (EVT), are the only approved treatments for acute ischemic stroke but must be administered in narrow therapeutic window of up to 4.5 and 6 hours, respectively. There were randomized controlled trials already published emphasizing the use of intravenous recombinant tissue plasminogen activator (IV rTPA) beyond the approved time frame in acute ischemic stroke. Important, 
in the pre-hospital setting, first responders should focus especially on the last time patients were known not to have stroke symptoms, as last known well. Advanced neuroimaging may help us overcome time constraints and expand the implementation of acute reperfusion therapies. Both computed tomography (CT) and Magnetic Resonance (MRI), provides valuable information in the management of patients with AIS, with diagnostic, therapeutic, and prognostic implications. The primary purposes of neuroimaging in patients with AIS are to determine the extent of initial ischemic core and identify the location and extent of intravascular clot as well as the presence and extent of penumbra, constituted by hypoperfused tissue at risk for infarction. Furthermore, over the past few years, advanced imaging protocols for acute stroke patients, mainly used in large institutions and comprehensive stroke centers, now include parenchymal imaging non-contrast head CT (NCT) or diffusion-weighted MRI (DWI) plus fluid attenuated inversion recovery (FLAIR) and Gradient echo MR imaging (GRE) or susceptibility-weighted imaging (SWI) on MRI, parenchymal imaging is meant to assess the volume of infarct. vascular imaging CT Angiography (CTA) or MR angiography (MRA), Vascular imaging is performed to detect the site of occlusion, and to characterize the collateral circulation. Penumbral imaging as well as Perfusion CT, Perfusion weighted imaging (PWI) aims at determining the volumes of salvageable penumbra [4]. This advanced neuroimaging helped overcome time constraints and expand the implementation of acute reperfusion therapies, for both EVT candidates in the late time window 6-24 hours and for IVT candidates 4.5-9 hours and wake-up patients [5]. There is no doubt that neuroimaging has played a crucial part in the assessment of patients with AIS over the past decades, as several parameters should be accurately individualized, either by CT, MR or angiography, to carefully select the patients that are eligible to IVT or IVT as outcome is dependent on that. The "2018 Guidelines for Management of Acute Ischemic Stroke” from the American Heart Association/American Stroke Association has a new recommendation that CT perfusion (CTP), diffusion-weighted imaging (DWI)-MRI, and/or MRI perfusion (MRP) be included as part of a standard imaging evaluation for patients within 6-24 h of symptom onset [6]. Thanks to recent RCTs, the therapeutic time window for both IVT and EVT in AIS has been extended, but also increased treatment algorithm complexity. In the event of AIS patients, acute reperfusion therapies with unknown time of symptom onset or in extended time windows are now possible. The present review provides an overview of the latest developments in the management of acute ischemic stroke, with an emphasis on thrombolytic therapy and intra-arterial intervention, summarizes the current evidence from randomized trials about its efficacy and safety of acute stroke care. Summarizes the current evidence from randomized trials about its efficacy and safety of acute stroke care, and that may assist clinicians in the selection of those late presenters that will most likely benefit from acute reperfusion therapies.

\section{Current concepts in intravenous thrombolysis treatment of acute ischemic stroke}

The intravenous administration of recombinant tissue plasminogen activator was introduced into acute stroke therapy in the mid-1990s [7-8], and there is no doubt that tPA improves outcome after stroke on AIS [9]. CT scan of the head has become routine in evaluating patients with signs and symptoms suggestive of AIS. Besides, pretreatment brain imaging with noncontrast CT is appropriate, mainly to exclude intracranial hemorrhage (ICH) and, to a lesser extent, reveal early ischemic changes. With the advent of thrombolysis, it becomes important to assess early ischemic changes in CT to predict the benefits of therapy. Regrettably, it is difficult to recognize and quantify these changes, which is why was born the Alberta stroke program early CT score (ASPECTS). The program was developed to offer the reliability and utility of a standard CT examination with a reproducible grading system to assess early ischemic changes, $<3$ hours from symptom onset, on pretreatment CT studies in patients with acute ischemic stroke of the anterior circulation [10]. ASPECTS is a systematic, robust, and practical method that can be applied to different axial baselines and is superior to that of the 1/3 MCA rule. ASPECTS is a 10-point scoring system that reliably predicts the extent of early ischemic changes from CT scans. At present, intravenous thrombolysis (IV rtPA) with $0.9 \mathrm{mg} / \mathrm{kg}$ alteplase, maximum dose $90 \mathrm{mg}$ over $60 \mathrm{~min}$ with initial $10 \%$ of dose given as bolus over $1 \mathrm{~min}$ ) is given to all patients that present within 4.5 hours of ischemic stroke independently of stroke etiology if all inclusion and exclusion criteria are fulfilled [11]. Certainly, that treatment with intravenous alteplase within 4.5 hours of acute ischemic stroke onset is associated with an increased early risk of intracerebral hemorrhage, but this risk is offset by later benefit in the form of reduced disability [12]. Since the 4.5 hours' time restriction is a major cause of IVT treatment failure, much effort has been made to select AIS patients who could safely undergo thrombolysis over extended time windows or with unknown time onset of symptoms. In fact, it's so that in recent years, the research focus has been on extending the time window for intravenous thrombolysis in acute ischemic stroke using new imaging techniques-based patient selection. Extending the time window for thrombolytic therapy is an important goal as it would increase the number of patients who are able to receive acute treatment. Consequently therefore, there have been attempts to expand time window for the benefit of additional patients presenting in the late hour window, thanks to new RCTs that have revolutionized acute ischemic stroke care by extending the use of intravenous thrombolysis therapy in time windows. Some trials have suggested that the treatment window may be extended in patients who are shown to have ischemic but not yet infarcted brain tissue on imaging. Trials that have suggested that the treatment window may be extended in patients who have been shown to have ischemic brain tissue but not yet infarcted on imaging are described below.

\section{The EXTEND trial}

Treatment with IV tPA outside the standard 4.5 hours window was evaluated by the Extending the Time for Thrombolysis in Emergency Neurological Deficits (EXTEND) trial. The trial was the first phase III randomized, placebo-controlled study to show benefit of IVT for patients presenting in the late hour window [13]. However, it must be premised that the rate of symptomatic intracerebral hemorrhage (sICH) in the EXTEND trial was among the highest reported. In this trial, thrombolysis was guided by perfusion imaging up to 9 hours after the onset of stroke. In effect in these cases advanced neuroimaging was used to select patients with CT perfusion or perfusion-diffusion 
MRI. The trial randomized 225 patients to either alteplase or placebo within 4.5 to 9.0 hours of symptoms onset or within 9 hours from the midpoint of sleep, if presenting with a wake-up stroke. Patients were included if they fulfilled three criteria: a) ischemic core $<70 \mathrm{ml}$, measured on CT perfusion as brain volume with cerebral blood flow $<30 \%$ of normal brain regions ( $\mathrm{rCBF}$ ) or on MRI as apparent diffusion coefficient (ADC) $<620 \mu \mathrm{m}^{2} / \mathrm{s}$, b) Critically hypoperfused brain volume $>10 \mathrm{ml}$ from ischemic core volume, measured on CT or magnetic resonance (MR) perfusion as delayed arrival of an injected racer agent (time to maximum of the residue function, Tmax $>6 \mathrm{~s}$ ), c) Perfusion lesion-ischemic core mismatch ratio $>1.2$. The primary outcome was a score of 0 or 1 on the modified Rankin scale, on which scores range from 0 (no symptoms) to 6 (death), at 90 days. The trials demonstrated that among the patients who had ischemic stroke and salvageable brain tissue, the use of alteplase between 4.5 and 9.0 hours after stroke onset or at the time the patient awoke with stroke symptoms resulted in a higher percentage of patients with no or minor neurologic deficits than the use of placebo. It showed that favorable outcome was more likely for the IV-rtPA group after adjustment for age and clinical severity at baseline, but not in unadjusted analysis. These results were obtained prior the early termination of the study, terminated due to loss of equipoise after publication of the WAKE-UP trial.

\section{EXTEND-IA TNK, EXTEND-IA TNK part 2 trial and NOR- TEST}

Current treatment with tissue plasminogen activator (tPA) requires an intravenous infusion of 1 hour because the clearance of tPA from the circulation is rapid ( $1 / 2$ approximately $6 \mathrm{~min}$ ). There have been multiple studies evaluating the safety and efficacy of alternative thrombolytic agents. Today, attention drawn to the benefits of intravenous (IV) tenecteplase (TNK) for acute stroke reperfusion therapy, particularly given the ease of administration and affordability. This thrombolytic medication is a genetically modified variant of alteplase with greater fibrin specificity and a longer half-life that permits bolus administration.

The Tenecteplase versus Alteplase before Endovascular Therapy for Ischemic Stroke (EXTEND-IA TNK) trial only involved patients with large vessel occlusions and compared $0.25 \mathrm{mg} / \mathrm{kg}$ of tenecteplase with standard dose IV-tPA [14]. The trial enrolled patients presenting within 4.5 hours of symptom onset that were eligible for mechanical thrombectomy, that is randomized tPA- and mechanical thrombectomy-eligible patients into tenecteplase and alteplase groups. The tenecteplase group had a higher incidence of reperfusion and better functional outcome, with similar adverse events before IVT than did intravenous alteplase. The recently concluded EXTENDIA TNK part-2 trial compared 2 different doses of TNK $(0.25 \mathrm{mg} /$ $\mathrm{kg}$ vs. $0.4 \mathrm{mg} / \mathrm{kg}$ ) prior to thrombectomy [15]. The trial authors randomly assigned patients with ischemic stroke who had occlusion of the internal carotid, basilar, or middle cerebral artery and who were eligible to undergo thrombectomy to receive tenecteplase (at a dose of $0.25 \mathrm{mg}$ per kilogram of body weight; maximum dose, 25 $\mathrm{mg}$ ) or alteplase (at a dose of $0.9 \mathrm{mg}$ per kilogram; maximum dose, $90 \mathrm{mg}$ ) within 4.5 hours after symptom onset. The results showed that a dose of $0.40 \mathrm{mg} / \mathrm{kg}$ of TNK, compared with $0.25 \mathrm{mg} / \mathrm{kg}$, did not significantly improve cerebral reperfusion in patients with large vessel occlusion ischemic stroke in whom endovascular thrombectomy is planned. The Tenecteplase versus alteplase for management of acute ischemic stroke (NOR-TEST) trial is the first randomized controlled phase 3 trial to investigate the safety and efficacy of tenecteplase in acute ischemic stroke, moreover the first clinical endpoint trial of tenecteplase at a high dose versus alteplase [16]. The study enrolled adults with suspected acute ischemic stroke who were eligible for thrombolysis and admitted within 4.5 hours of symptom onset or within 4.5 hours of awakening with symptoms, or who were eligible for bridging therapy before thrombectomy. A total of 1100 patients who fulfilled standard thrombolysis eligibility criteria and were randomly assigned; the conventional primary endpoint of excellent outcome, defined by scores 0 to 1 occurred in 354 (64\%) patients in the tenecteplase group and 345 (63\%) patients in the alteplase group at 3 months. The frequency of symptomatic intracerebral hemorrhage was similar between groups. The study failed to test the hypothesis under investigation that is whether tenecteplase is superior to alteplase, due to insufficient statistical power from the expectation of a large treatment effect, and why about $80 \%$ of participants present with mild neurological damage, a transient ischemic attack, or a mimic of stroke. However, the NOR-TEST investigators proved the safety and efficacy of using a dose of $0.4 \mathrm{mg} / \mathrm{kg}$ of tenecteplase with similar rates of symptomatic intracerebral hemorrhage and functional independence when compared with IV-tPA. It should be noted that the use of tenecteplase for the treatment of acute stroke is currently off label.

\section{WAKE-UP TRIAL}

In 14 to $27 \%$ of strokes, the time of symptom onset is not known, frequently because stroke symptoms are recognized when the patient awakes from sleeping [17-18]. Therefore, many patients with stroke are precluded from thrombolysis treatment because the time from onset of their symptoms is unknown. For this reason, different advanced neuroimaging MRI sequences sensitive to different aspects of tissue pathophysiology in acute cerebral ischemia have been used. DWI/FLAIR mismatch has been shown to predict stroke onset $<4.5 \mathrm{~h}$ with a sensitivity of $78 \%$ and positive predictive value of $83 \%$, even up to $87 \%$ for middle cerebral artery (MCA) [19]. Using this concept, in another example of expanding indications for IV thrombolysis, the Efficacy and Safety of MRI-Based Thrombolysis in Wake-Up Stroke (WAKE-UP) trial showed that patients who presented within 4.5 hours of awakening with stroke symptoms had a small benefit with IV-tPA if MRI DWI showed a diffusion-restricting lesion without matching hyperintense signal on fluid attenuated inversion recovery (FLAIR) sequence [20]. In these trial patients with wake-up stroke (WUS) with unknown symptom onset time or patients that woke up with symptoms, presenting within 4.5 hours from awakening with positive DWI MRI of the brain and absence of signal FLAIR imaging were randomized to either IVT with alteplase $0.9 \mathrm{mg} / \mathrm{kg}$ or placebo. AIS patients treated with IVT had higher rates of 3-month favorable functional outcome FFO) $53 \%$ compared to placebo $42 \%$ despite a greater risk for parenchymal hematoma. The WAKE-UP trial protocol paved the way in expanding the indications for safe and effective 
delivery of IV-tPA, but it was not aimed at extending the time window of the IVT. The demonstration of a "mismatch" between an abnormal diffusion-weighted imaging (DWI) signal and a normal fluidattenuated inversion recovery (FLAIR) on brain MRI plays a central role in the new guidelines. So much so that the recommendations from the American Heart Association / American Stroke Association (AHA / ASA) represented a marked change in the role of imaging for AIS, particularly for "awakening" stroke, guidelines updated in 2019 to reflect new data favoring the use of the DWI-FLAIR mismatch as a decision-making tool for stroke upon awakening [21]. However, they are "moderate" recommendation for alteplase treatment guided by magnetic resonance imaging (MRI) in patients with unknown time of symptom onset.

Randomized, Multicenter Trial of ARTSS-2, and multimodal CT or MRI for IV thrombolysis in ischemic stroke with unknown time of onset.

Interestingly, a recent phase-II randomised controlled trial ARTSS-2 (Argatroban With Recombinant Tissue Plasminogen Activator for Acute Stroke) assessing the adjunctive use of a direct thrombin inhibitor (argatroban) in addition to IVT shows no increase in the risk of sICH. ARTSS-2 is the first randomized trial of concurrent IV thrombolysis and anticoagulation [22]. In this trial, patients treated with standard-dose r-tPA, not receiving endovascular therapy, were randomized to receive no argatroban or argatroban (100 $\mu \mathrm{g} / \mathrm{kg}$ bolus) followed by infusion of either 1 (low dose) or $3 \mu \mathrm{g} / \mathrm{kg}$ per minute (high dose) for 48 hours. Safety was incidence of symptomatic intracerebral hemorrhage. Probability of clinical benefit (modified Rankin Scale score 0-1 at 90 days) was estimated using a conservative Bayesian Poisson model (neutral prior probability centered at relative risk, 1.0 and 95\% prior intervals, 0.33-3.0) Despite the limited number of patients studied, conservative Bayesian analyses indicated a $79 \%$ probability that adjunctive argatroban, a direct thrombininhibitor, increased the percent of patients with a score of $0-1$ on the modified Rankin scale (the scale runs from 0-6 with "0" being perfect health without symptoms to "6" being death. 0 - No symptoms) at 90 days. Macha et al [23]. Compared 2 different protocols to enable thrombolysis in the extended or unknown time window after stroke onset with either multimodal CT or MRI. IV thrombolysis was performed in 100 patients $(54.3 \%)$ based on multimodal CT imaging and in 84 patients (45.7\%) based on MRI, with evidence of salvageable brain tissue at risk on CT or MR perfusion imaging (mismatch between hypoperfusion versus infarcted core, mismatch quotient $>1.4$ ), and unknown time of onset and $>4.5$ hours from last known well, including patients with wake-up stroke and patients with known time of onset $>4.5$ hours (unknown and extended window, respectively), with no upper limit of time, is feasible, with safety and efficacy outcomes comparable to previous randomized trials. The authors reported that IV thrombolysis in IAS in the unknown or extended time window appeared safe in CT and MRI selected patients, while the use of CT imaging led to faster door-to-needle times.

\section{Current concepts in endovascular treatment of acute ischemic stroke}

Until recently, intravenous recombinant tissue-type plasminogen activator was the only evidence-based treatment option. In general, but terminology may vary, acute occlusion of the intracranial internal carotid artery (ICA), proximal posterior cerebral artery, middle cerebral artery (MCA), anterior cerebral arteries, and/or basilar artery are commonly referred to as large vessel occlusions (LVOs). This class of AIS has larger infarct sizes, more severe presentation deficits, and worse clinical outcomes, so it is conceivable that the endovascular EVT may disproportionately benefit stroke-related dependence and death [24]. However, the past several years has witnessed dynamic developments in the field of EVT with respect to AIS, to the point of prove that EVT is effective in treating ischemic strokes due to large vessel occlusion (LVO). Only after the 2015 publication of five clinical trials, EVT has become the standard of care in patients with acute ischemic stroke with large-vessel occlusion (LVO) presenting within 6 hours from symptom onset. The five studies (MR CLEAN, ESCAPE, REVASCAT, SWIFT PRIME and EXTEND IA) were conducted between December 2010 and December 2014 [25]. These studies collectively established the overall safety and efficacy of interventional endovascular treatment for acute ischemic stroke. The HERMES (Highly Effective Reperfusion evaluated in Multiple Endovascular Stroke Trials) collaboration was formed to pool patient data from these 5 trials. The HERMES trial concluded that EVT reduced disability from anterior circulation stroke with LVO, and benefits could be seen in most patients, irrespective of patient characteristics including age or geographical locations. Furthermore, from the meta-analysis and other studies, we know that overall, the risk of reperfusion hemorrhage is relatively low, $4.4 \%$ in the EVT arm versus $4.3 \%$ in the control arm [26]. Guidelines for Management of Acute Ischemic Stroke from the American Heart Association/American Stroke Association has a new recommendation that $\mathrm{CT}$ perfusion (CTP), diffusion-weighted imaging (DWI)-MRI, and/or MRI perfusion (MRP) be included as part of a standard imaging evaluation for patients within 6-24 h of symptom onset [6].

DAWN (DWI or CTP Assessment with Clinical Mismatch in the Triage of Wake-Up and Late Presenting Strokes Undergoing Neurointervention with Trevo) trial

This multi-centre, prospective, open-label trial with blinded outcome assessment trial proved the efficacy of EVT up to $24 \mathrm{~h}$ after symptom onset [27]. Patients with LVO in the anterior circulation on CTA or magnetic resonance angiogram and who had a determined mismatch between the radiological core infarct and clinical deficits, that is clinical-core mismatch, were randomized to EVT or conservative treatment. It should be noted that the MRI or CT perfusion with the use of RAPID software was only used to estimate infarct core. Indeed, in these trials, the need for fast and accurate interpretation of brain and vascular imaging from the emergency radiologist has never been more important, by identifying patients with a relatively small core infarct, the radiologist can assist the stroke neurologist in selecting patients who are most likely to benefit from EVT. The DAWN study's included patients with MCA and/or ICA occlusion presenting within 6-24 hours of last known well with NIHSS scores of 10 or more and a relatively small volume of core infarct as assessed by diffusionweighted MRI or perfusion CT, ideally less than $30 \mathrm{~cm}^{3}$. Effectively, the aim was to establish whether subjects considered to have substantial areas of salvageable brain based on age-adjusted clinical 
core mismatch who can undergo endovascular treatment within 6-24 $\mathrm{h}$ from time last seen well (TLSW) have better outcomes at three months compared to subjects treated with standard medical therapy alone. Age-adjusted clinical core mismatch is defined by age; indeed, patients were stratified into 3 groups: Group $\mathrm{A} \geq 80$ years of age, baseline National Institutes of Health Stroke Scale NIHSS $\geq 10$, and infarct volume $<21 \mathrm{~cm}^{3}$; Group B $<80$ years of age, NIHSS $\geq 10$, and infarct volume $<31 \mathrm{~cm}^{3}$; and Group C $<80$ years of age, NIHSS $\geq 20$, infarct volume 31 to $<51 \mathrm{~cm}^{3}$. The results of the reported DAWN trial were highly in favor of EVT. Pre-specified interim analysis indicated a high probability of benefit with thrombectomy over standard medical management, resulting in early termination of the trial, indeed, 3-month functional independence: $49 \%$ in the EVT group versus $13 \%$ in the best medical management group) and analogous to the positive results of early time window EVT trials. In summary, the time window for endovascular treatment may be extended to 24 hours after the patient was last known to be well if patients are carefully selected based on a disproportionately severe clinical deficit in comparison with the size of the stroke on imaging.

DEFUSE 3 (The Endovascular Therapy Following Imaging Evaluation for Ischemic Stroke)

Concurrently, another multicenter, randomized, open-label trial, with blinded outcome assessment, commonly named Endovascular Therapy Following Imaging Evaluation for Ischemic Stroke (DEFUSE 3), examined the outcomes for endovascular intervention for a similar population of patients with anterior circulation LVO between 6 and 16 hours of last known well, with slightly distinct qualifying criteria [28]. While the DAWN trial used a selection paradigm that assigned a pre-treatment core infarct threshold (maximum of 50ml) based on patient age and presenting NIHSS, DEFUSE 3 trial was more inclusive. In this trial, 182 patients, between 6 and 16 hours after the time last known well, had been randomized to thrombectomy plus medical therapy versus medical therapy alone, prior to early trial termination for efficacy. Patients were eligible if they had an initial infarct volume of less than $70 \mathrm{ml}$, a ratio of volume of ischemic tissue to initial infarct volume of 1.8 or more, and an absolute volume of potentially reversible ischemia of $15 \mathrm{ml}$ or more, assessed from CT perfusion or MR diffusion. Importantly, DEFUSE3 included patients with NIHSS between 6 and 9 in addition to those with NIHSS $>10$, patients over 90 years old, and those with baseline mRS of 2. Ultimately, in this trial, endovascular thrombectomy for ischemic stroke 6 to 16 hours after a patient was last known to be well plus standard medical therapy resulted in better 90-day functional outcomes than standard medical therapy alone among patients who had evidence of salvageable tissue based on a formula that incorporated early infarct size and the volume of hypoperfused tissue on perfusion imaging

\section{Endovascular treatment of acute ischemic stroke in posterior circulation}

Posterior circulation strokes are known to lead to worse clinical outcomes compared to strokes in the anterior circulation [29]. Basilar Artery Occlusion (BAO), which represent most of the posterior circulation LVO, tend to have devastating outcomes. The basilar artery is generally the primary artery that supplies blood flow to the posterior circulation including the brain stem, occipital lobes, and part of the cerebellum and thalami. As the territory of the posterior circulation is very small compared with anterior circulation, even a small infarct can lead to life-threatening complications. It carries a high mortality of $85-95 \%$ if recanalization does not occur, and a substantial part of survivors suffer severe disability, some being in locked in state [30]. Despite predominant evidence for endovascular therapy in anterior circulation ischemic stroke due to large-vessel occlusion, data regarding the treatment of acute $\mathrm{BAO}$ are still equivocal, especially regarding revascularization therapies. The recent trials excluded patients with posterior circulation LVO. Intravenous thrombolysis is the conventional standard-of-care in eligible patients with acute BAO and other strokes of the posterior circulation; however, thrombolysis alone may not yield satisfactory reperfusion in many patients in acute $\mathrm{BAO}$, pharmacological thrombolysis, was adjuncted or replaced with invasive, endovascular thrombectomy procedures. It is widely agreed that meaningful survival after BAO requires rapid access to revascularization, but a considerable proportion of successful recanalizations do not translate into clinical benefit; this defines the term "futile recanalization". Futile recanalization was defined as successful recanalization with no clinical benefit demonstrated as an mRS score $4-6$ at 3 months. There is abundant, data from the registries and retrospective series showing benefit from mechanical thrombectomy (MT), with successful reperfusion in selected patients, most notably to exclude victims of already extended ischemia, would assist in translating excellent recanalization rates into improved clinical outcomes and more acceptable futility rates. Currently, treatments using intravenous thrombolytic agents or intra-arterial treatments for BAO remain unclear of its efficacy whereas mechanical thrombectomy (MT) is thought to be the most effective treatment. About this a recent large meta-analysis of 1,358 patients showed MT is the most effective method of treatment of acute basilar artery occlusion. The efficacy of IV or IA thrombolytic therapy in BAO remains unclear; despite now the 'gold-standard' method of revascularization has not been definitively established, evidence strongly favor MT as the most effective treatment [31]. A recent systematic review comparing intravenous thrombolysis with mechanical thrombectomy suggests that the introduction of mechanical thrombectomy techniques, including aspiration and stent retriever thrombectomy, resulted in improved clinical and angiographic outcomes and safety profile. Therefore, we can conclude that endovascular mechanical approaches have been reported to provide superior outcomes over pharmacological thrombolysis in basilar artery occlusion [31]. The REVASK (Revascularization in Ischemic Stroke Patients) registry from Germany, suggests that MT in posterior circulation stroke (PCS) shows a lower risk of symptomatic intracranial hemorrhage and similar effectiveness compared to anterior circulation stroke (ACS). PCS patients also seem to benefit from MT started beyond 6 $\mathrm{h}$ after symptom onset. In any case mechanical thrombectomy (MT) following intravenous administration of IV-rt-PA is considered an effective treatment for the occlusion of the internal carotid artery or the M1 segment of the middle cerebral artery. 


\section{The Clinical Usefulness Hypothermia after Endovascular Thrombectomy}

Despite the remarkable achievement of EVT from acute ischemic stroke with emergent large-vessel occlusion, many stroke patients still experience disabilities despite the high reperfusion rate, as in the case of characteristic stroke involving the entire middle cerebral artery (MCA) territory, also 'malignant MCA infarction', can be catastrophic with a mortality rate of up to $70 \%$ [33]. Advances in thrombectomy techniques have led to a high rate of reperfusion for carotid terminus occlusions. With these improvements in EVT outcomes, ICA terminus occlusions can now usually be categorized along with MCA M1 occlusions under the term anterior circulation LVO. However, there is still a population where outcomes are universally grave despite best EVT. The potential benefit of EVT is likely less and the risks of hemorrhage greater in patients who present late or have very large core sizes $(>100 \mathrm{~mL})$ [34]. These patients may be candidates for combining EVT with future therapeutic advances, such as hypothermia. Indeed, targeted temperature management (TTM) may be more beneficial after endovascular treatment (EVT) in patients with a large ischemic core [35]. These results may provide useful direction in the design of future clinical trials.

\section{Conclusions}

The recent advancements in EVT and IVT have revolutionized treatment of AIS. The two therapies constitute in combination the standard of care for patients with acute ischemic stroke with anterior circulation large vessel occlusion. The therapeutic window has shown an expansion in recent years for these two therapies, evaluating various mismatches using advanced imaging in AIS. Still, the status of AIS reperfusion treatments in extended time windows is dominated by high-quality data from RCTs that used advanced neuroimaging (CT/MR perfusion or MRI) to select patients. The recanalization therapies in late time windows are not investigational nor a luxury for modern acute stroke care. Far from it, they are life-saving treatment modalities for a major cause of disability and among the leading causes of mortality worldwide, and their widespread use in clinical practice is an urgent need. New treatment algorithms for AIS need for AIS in extended time windows, and advanced neuroimaging capability acquisition in stroke centers, which is not an easy task. Further clinical trials are underway to broaden the horizon of acute stroke treatment, and we must also aim to have even more advanced imaging to achieve a broader therapeutic window and better clinical outcomes soon. Timely reperfusion in AIS is the most effective treatment available, new schemes and patients await us for which interventions maybe applied. We wait that advanced imaging focused on assessing collateral circulation and cerebral hemodynamics can help select patients who are most likely to benefit from IVT and EVT, even beyond the times studied to date. Advanced imaging focused on assessing collateral circulation and cerebral hemodynamics can help select patients who are most likely to benefit from IVT and MT, even beyond the times studied to date Finally, I think the key challenge that we will face is the organization of stroke care which aims to get the right patient to the right hospital as fast as possible.

\section{References}

1. Song S, Liang L, Fonarow GC, Smith EE, Bhatt DL, et al. (2019) Comparison of Clinical Care and In-Hospital Outcomes of Asian American and White Patients With Acute Ischemic Stroke. JAMA Neurol 76: 430-439. [crossref]

2. Mozaffarian D, Benjamin EJ, Go AS, Arnett DK, Blaha MJ, et al. (2016) Heart disease and stroke statistics-2016 update: a report from the American Heart Association. Circulation 133: 38-360. [crossref]

3. Rowley HA (2005) Extending the time window for thrombolysis: evidence from acute stroke trials. Neuroimaging Clin North Am 15: 575-587. [crossref]

4. Shams M, Shams S, Wintermark M (2020) What's new in imaging of acute stroke? Intensive Care Med 46: 1453-1456. [crossref]

5. Nogueira RG, Jadhav AP, Haussen DC, Bonafe A, Budzik RF, et al. (2018) Thrombectomy 6 to 24 hours after stroke with a mismatch between deficit and infarct. N Engl J Med 378: 11-21.

6. Powers WJ, Rabinstein AA, Ackerson T, Adeoye OM, Bambakidis NC, et al. (2018) 2018 Guidelines for the Early Management of Patients With Acute Ischemic Stroke: A Guideline for Healthcare Professionals From the American Heart Association/ American Stroke Association. Stroke 49: 46-110. [crossref]

7. Thomalla G, Rossbach P, Rosenkranz M, Siemonsen S, Krützelmann A, et al. (2009) Negative fluid-attenuated inversion recovery imaging identifies acute ischemic stroke at 3 hours or less. Ann Neurol 65: 724-732. [crossref]

8. Aoki J, Kimura K, Iguchi Y, Shibazaki K, Sakai K, et al. (2010) FLAIR can estimate the onset time in acute ischemic stroke patients. J Neurol Sci 293: 39-44. [crossref]

9. National Institute of Neurological Disorders and Stroke rt-PA Stroke Study Group (1995) Tissue plasminogen activator for acute ischemic stroke. N Engl J Med 333: 1581-7. [crossref]

10. Barber PA, Demchuk AM, Zhang J, Buchan AM (2000) Validity and reliability of a quantitative computed tomography score in predicting outcome of hyperacute stroke before thrombolytic therapy. ASPECTS Study Group. Alberta Stroke Programme Early CT Score. Lancet 355: 1670-4. [crossref]

11. Powers WJ, Rabinstein AA, Ackerson T, Adeoye OM, Bambakidis NC, et al (2019) Guidelines for the early management of patients with acute ischemic stroke: 2019 update to the 2018 guidelines for the early management of acute ischemic stroke: a guideline for healthcare professionals from the American Heart Association/ American Stroke Association. Stroke 50: 344-418. [crossref]

12. Emberson J, Lees KR, Lyden P, Blackwell L, Albers G, et al. (2014) Effect of treatment delay, age, and stroke severity on the effects of intravenous thrombolysis with alteplase for acute ischaemic stroke: a meta-analysis of individual patient data from randomised trials. Lancet 384: 1929-35. [crossref]

13. Seners P, Turc G, Maïer B, Mas JL, Oppenheim C, et al. (2016) Incidence and predictors of early recanalization after intravenous thrombolysis: a systematic review and meta-analysis. Stroke 47: 2409-2412. [crossref]

14. Campbell BCV, Mitchell PJ, Churilov L, Yassi N, Kleinig TJ, et al. (2018) Tenecteplase versus Alteplase before Thrombectomy for Ischemic Stroke. N Engl J Med 378: 15731582 .

15. Campbell BCV, Mitchell PJ, Churilov L, Yassi N, Kleinig TJ, et al. (2020) Effect of Intravenous Tenecteplase Dose on Cerebral Reperfusion Before Thrombectomy in Patients With Large Vessel Occlusion Ischemic Stroke: The EXTEND-IA TNK Part 2 Randomized Clinical Trial. JAMA 323: 1257-1265. [crossref]

16. Logallo N, Novotny V, Assmus J, Kvistad CE, Alteheld L, et al. (2017) Tenecteplase versus alteplase for management of acute ischaemic stroke (NOR-TEST): a phase 3 , randomised, open-label, blinded endpoint trial. Lancet Neurol 16: 781-788. [crossref]

17. Fink JN, Kumar S, Horkan C, Linfante I, Selim MH, et al. (2002) The stroke patient who woke up: clinical and radiological features, including diffusion and perfusion MRI. Stroke 33: 988-993. [crossref]

18. Mackey J, Kleindorfer D, Sucharew H, Moomaw CJ, Kissela BM, et al. (2011) Population-based study of wake-up strokes. Neurology 76: 1662-1667. [crossref]

19. Aoki J, Kimura K, Iguchi Y, Shibazaki K, Sakai K, et al. (2010) FLAIR can estimate the onset time in acute ischemic stroke patients. J Neurol Sci 293: 39-44. [crossref]

20. Thomalla G, Simonsen CZ, Boutitie F, Andersen G, Berthezene Y, et al. (2018) MRIguided thrombolysis for stroke with unknown time of onset. N Engl J Med 379: 611- 622. 
21. Powers WJ, Rabinstein AA, Ackerson T, Adeoye OM, Bambakidis NC, et al. (2019) Guidelines for the early management of patients with acute ischemic stroke: 2019 Update to the 2018 Guidelines for the Early Management of Acute Ischemic Stroke: a guideline for healthcare professionals from the American Heart Association/ American Stroke Association. Stroke 50: 344-418. [crossref]

22. Barreto AD, Ford GA, Shen L, Pedroza C, Tyson J, et al. (2017) Randomized, multicenter trial of ARTSS-2 (Argatroban with recombinant tissue plasminogen activator for acute stroke). Stroke 48:1608-16. [crossref]

23. Macha K, Hoelter P, Siedler G, Knott M, Schwab S, et al. (2020) Multimodal CT or MRI for IV thrombolysis in ischemic stroke with unknown time of onset. Neurology 95: 2954-2964. [crossref]

24. Malhotra K, Gornbein J, Saver JL (2017) Ischemic Strokes Due to Large-Vessel Occlusions Contribute Disproportionately to Stroke-Related Dependence and Death: A Review. Front Neurol 8: 651. [crossref]

25. Hong JM, Lee JS, Song HJ, Jeong HS, Choi HA, et al. (2014) Therapeutic hypothermia after recanalization in patients with acute ischemic stroke. Stroke 45: 134-40. [crossref]

26. Sharobeam A, Jones B, Walton-Sonda D, Lueck CJ (2021) Factors delaying intravenous thrombolytic therapy in acute ischaemic stroke: a systematic review of the literature. J Neurol 268: 2723-2734. [crossref]

27. Nogueira RG, Jadhav AP, Haussen DC, Bonafe A, Budzik RF, et al. (2018) Thrombectomy 6 to 24 hours after stroke with a mismatch between deficit and infarct. New Engl J Med 378: 11-21.

28. Albers GW, Marks MP, Kemp S, Christensen S, Tsai JP, et al. (2018) Thrombectomy for Stroke at 6 to 16 Hours with Selection by Perfusion Imaging. N Engl J Med 378: 708-718. [crossref]
29. Sommer P, Posekany A, Serles W, Marko M, Scharer S, et al. (2018) Is Functional Outcome Different in Posterior and Anterior Circulation Stroke? Stroke 49:27282732. [crossref]

30. Lindsberg PJ, Sairanen T, Strbian D, Kaste M (2012) Current treatment of basilar artery occlusion. Ann NY Acad Sci 1268: 35-44. [crossref]

31. Wyszomirski A, Szczyrba S, Tomaka D, Karaszewski B (2017) Treatment of acute basilar artery occlusion: systematic review and meta-analysis. Neurol Neurochir Pol 51: 486-96. [crossref]

32. Weber R, Minnerup J, Nordmeyer H, Eyding J, Krogias C, et al. (2019) Thrombectomy in posterior circulation stroke: differences in procedures and outcome compared to anterior circulation stroke in the prospective multicentre REVASK registry. Eur J Neurol 26:299-305. [crossref]

33. Hacke W, Schwab S, Horn M, Spranger M, De Georgia M, et al. (1996) 'Malignant' middle cerebral artery territory infarction: clinical course and prognostic signs. Arch Neurol 53: 309-15. [crossref]

34. Sarraj A, Hassan AE, Savitz S, Sitton C, Grotta J, et al. (2019) Outcomes of Endovascular Thrombectomy vs Medical Management Alone in Patients With Large Ischemic Cores: A Secondary Analysis of the Optimizing Patient's Selection for Endovascular Treatment in Acute Ischemic Stroke (SELECT) Study. JAMA Neurol 76: 1147-1156. [crossref]

35. Choi MH, Gil YE, Lee SJ, Lee JS, Hong JH, et al. (2021) The clinical usefulness of targeted temperature management in acute ischemic stroke with malignant trait after endovascular thrombectomy. Neurocrit Care 34: 990-999. [crossref]

\section{Citation:}

Rocco Galimi (2021) Recent Updates on Intravenous Thrombolysis and Endovascular Theraphy for Hyperacute Manegement of Ischemic Strokes. J Neurol Neurocrit Care Volume 4(3): 1-7. 